Commun: Communication et organisation

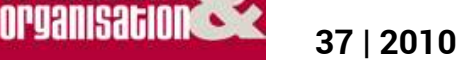

La communication à l'épreuve des mutations économiques

\title{
L'activité langagière située au risque des TIC
}

Le cadre d'analyse est-il toujours pertinent pour les organisations?

Isabelle Comtet

(2) OpenEdition

Journals

Édition électronique

URL : http://journals.openedition.org/communicationorganisation/1298

DOI : 10.4000/communicationorganisation. 1298

ISSN : $1775-3546$

Éditeur

Presses universitaires de Bordeaux

Édition imprimée

Date de publication : 1 juin 2010

Pagination : $125-138$

ISBN : 978-2-86781-710-6

ISSN : $1168-5549$

Référence électronique

Isabelle Comtet, "L'activité langagière située au risque des TIC », Communication et organisation [En

ligne], 37 | 2010, mis en ligne le 02 mai 2011, consulté le 19 avril 2019. URL : http://

journals.openedition.org/communicationorganisation/1298; DOI : 10.4000/

communicationorganisation. 1298

(c) Presses universitaires de Bordeaux 


\title{
L' activité langagière située au risque des TIC. Le cadre d'analyse est-il toujours pertinent pour les organisations?
}

\author{
Isabelle Comtet $^{1}$
}

\section{Introduction}

Le thème de l'usage des dispositifs informatiques professionnels permettant une communication plurielle médiatisée (type groupware) n'est pas un sujet nouveau lié au développement d'un secteur d'activité en particulier. Pour les entreprises, l'usage de tels dispositifs, en contexte de mondialisation, a pour objectif de maintenir ou d'accroître leur capacité de production en développant de nouvelles formes d'activités, coordonnées par les dispositifs techniques. Notre travail se centre sur la prise en compte du changement technologique par les acteurs en jeu dans cette situation et sur l'appropriation de ce type de technologie dans une démarche d'amélioration du processus communicationnel. Il s'agit de penser le rapport aux TIC comme un construit social.

Or, ce construit social ne peut s'élaborer qu'en considérant qu'il est le fruit d'un processus dans lequel le langage joue un rôle central: ce dernier permet de préciser, de définir, de comprendre et donc, finalement, de produire un contexte d'action individuelle (bien qu'immergé dans le collectif) dans lequel l'acteur réussit à s'approprier les TIC. Il est le vecteur des pratiques organisationnelles. C'est pourquoi il est consubstantiel à un usage professionnel efficace des TIC. L'intérêt de considérer l'analyse de la situation dans une perspective différente de celle de l'innovation ou de la diffusion technologique est de mettre l'accent sur le rôle des acteurs dans le processus-même du développement d'un usage dans un contexte particulier.

On ne se situe pas ici en amont de l'usage des TIC comme peuvent le faire la plupart des concepteurs de dispositifs ${ }^{2}$, ni en aval comme en rendent 01. Isabelle Comtet est Maître de Conférences en Sciences de l'Information et de la Communication à l'Université Jean Moulin Lyon 3. Elle est chercheur à SICOMOR, Groupe de recherche en Système d'information et communication des organisations, dans cette Université. Mail : isabelle.comteł@univ-lyon3.fr 02. Quoique se développent aussi des travaux sur la " conception assistée par l'usage » comme ceux de P. Mallein (CNRS-Grenoble) 
compte certaines études sociologiques ou informatiques. On est au contraire au cour du problème de l'émergence d'un changement de mode de travail. Il existe donc un lien fort entre les TIC et les acteurs professionnels qui les investissent.

\section{Usages des Technologies collaboratives et communication langagière : quelle problématique pour les organisations?}

Le travail dit "collaboratif » distant implique une interaction durable au cours de laquelle l'information est traitée et discutée, pour arriver à un accord qui permette de mettre en place et de mener à bonne fin la réalisation d'une activité commune. Dès la construction de ce dispositif alliant acteurs et technologies, la pérennité et la solidité de l'interaction, gages d'efficacité au travail, exigent des modifications organisationnelles, sociales, et managériales. Les pratiques organisationnelles doivent donc évoluer dans le même temps.

En effet, une telle orientation de la problématique implique de porter la réflexion sur les usagers en tant qu'acteurs sociaux ${ }^{3}$ au sein de l'entreprise: le travail sera-t-il plus collectif? La communication sera-t-elle plus étendue (c'est-à-dire multi-personnelle ou seulement bilatérale)? La communication interpersonnelle sera-t-elle facilitée par les TIC? Les liens interpersonnels auront-ils un caractère de permanence ou d'immédiateté selon les TIC utilisées? Et surtout, ce travail collaboratif sera-t-il performant?

On est conduit à prendre en compte plusieurs paramètres du contexte:

- les caractéristiques intrinsèques de l'outil (synchrone, asynchrone, quasisynchrone),

- les pratiques existantes des utilisateurs (usages de la vidéo, du Web, des applications partagées etc..),

- les conditions de réalisation de l'activité elle-même (lieu de réalisation, temps de travail, nombre d'interactants, pratiques organisationnelles, etc.),

- et la nature de cette dernière (travail en mode "projet", relecture, élaboration d'un travail véritablement collaboratif ${ }^{4}$, etc.).

Dès lors, il est essentiel pour les organisations de comprendre comment d'une part, ces usages modifient le processus communicationnel grâce auquel des acteurs travaillent ensemble et, d'autre part, comment ils infléchissent la réalisation de l'activité. Or, dans la communication organisationnelle, nous l'avons souligné, l'activité langagière joue un rôle primordial, notamment au

03. Par " acteur social », nous entendons un acteur qui appartient à un groupe et participe de ses caractères. 04. Dans le travail de groupe, nous savons qu'une activité professionnelle peut être individuelle, ou supposer le travail de plusieurs personnes. Il y a là une certaine granularité, une progression par valeurs successives : I'activité peut être fragmentée en différentes formes organisationnelles; celles-ci s'ajoutent les unes aux autres (coordination, puis coopération impliquant la coordination) générant différents degrés de travail collectif. Cette granularité est elle-même induite par le dispositif sociotechnique utilisé pour la réalisation du travail. La possibilité de réaliser une activité plurielle (nous prenons « plurielle » dans l'acception la plus usuelle qui est celle d'une activité impliquant un nombre de personnes supérieur à une unitél, ou une activité collective (nous utilisons le terme « collectif » pour évoquer des personnes constituées en groupe), ou une activité collaborative (impliquant un travail conjoint). 
cours de l'appropriation: elle est présente dans les caractéristiques-mêmes des outils (vidéoconférence par exemple); elle est le support de pratiques professionnelles (avec le courriel par exemple); elle est un paramètre incontournable des conditions de réalisation de l'activité (travail multilingue, par exemple) et elle influence la nature du travail lui-même (ainsi le travail par négociation).

\section{Évolution des pratiques organisationnelles et langage: quels enjeux méthodologiques?}

Le centrage sur l'articulation entre usage des technologies collaboratives et communication langagière amène à rendre compte de façon nettement marquée du contexte d'action individuelle qui recouvre le rapport de l'acteur à l'outil. Notamment pris sous l'angle de l'appropriation, le contexte d'action individuelle transite par l'activité langagière. Cette dimension est souvent laissée de côté au profit de l'unique prise en considération du contexte d'action collective qui concerne le fonctionnement du groupe dans l'organisation. Cette problématique "augmentée» suppose de mettre en relation trois dimensions différentes de l'activité. La première met l'accent sur l'évolution des outils pour la réalisation du travail collaboratif; la seconde, en lien avec la première, concerne la reconfiguration des liens sociaux pour la réalisation de l'activité au sein des organisations aujourd'hui. La troisième met l'accent sur la nécessaire modification du travail et sur la capacité qu'a la communication langagière à faciliter ces nouveaux modes d'activité.

\section{Co-construction technologique et organisationnelle?}

Intrinsèquement, les dispositifs sociotechniques collaboratifs - ou groupwares - sont centrés sur les groupes et les processus qui s'y déploient. Il en existe différents types dont les objectifs et les caractéristiques diffèrent. En fonction des configurations de travail, les systèmes de travail collaboratif se présentent sous diverses formes, par exemple, des groupwares asynchrones pour faciliter la gestion des flux électroniques ou des groupwares synchrones pour favoriser une création en temps réel. Ce type de technologie a donc pour finalité la création d'un espace de travail partagé matériel et virtuel, soustendu par une collaboration dynamique dans un groupe de travail, au-delà des contraintes spatio-temporelles. Dès lors, les systèmes de travail collaboratif peuvent être considérés comme de réels outils d'aide à la structuration des activités professionnelles d'un collectif de travail (Vacherand-Revel, 2001). Dans le même ordre d'idée, pris sous l'angle des liens qui unissent les acteurs, on sait que les systèmes de travail collaboratif induisent un système d'échanges régulés qui génère des dépendances informationnelles et cognitives mutuelles (Finholt, Sproull, Kiesler, 1995) ${ }^{5}$. Celles-ci doivent, logiquement, stimuler une

05. Finholt T., Sproull L., Kiesler S., 1995, Communication and performance in Ad Hoc task groups, in Intellectual Team Work, LEA, New Jersey 
coopération $^{6}$ entre les individus. L'objectif est alors d'améliorer le rendement au travail en croisant les contextes d'actions individuelles et collectives.

Cherchant à répondre aux besoins organisationnels, les groupwares offrent aujourd'hui un spectre de fonctionnalités de plus en plus large et ont été englobés dans ce qu'on appelle les environnements de travail collaboratifs (Collaborative Working Environment). Ces derniers sont composés de différents outils allant de la messagerie électronique à la vidéoconférence, en passant par le workflow ou la gestion électronique de document (Membrado, 2008) $)^{7}$. Ce sur quoi est mis l'accent, c'est la nécessité de faire évoluer les modalités de communication en fonction du contexte socio-économique dans lequel les TIC s'insèrent et en fonction des pratiques professionnelles. Ces dernières sont le reflet de la confrontation permanente existant entre le contexte d'action collective (qui est celui de l'acteur au sein de l'organisation) et le contexte d'action individuelle (qui est celui de l'acteur dans son rapport à l'outil).

Dorénavant, on observe, dans l'environnement de travail collaboratif des outils qui favorisent une collaboration "mixte ». Cette dernière est soustendue par l'usage d'outils synchrones (comme la vidéo conférence), en même temps que d'outils quasi-synchrones (comme le chat) et que d'outils asynchrone (comme le mail). Cette évolution est semblable à celle des technologies web. Par exemple, «Le Metaweb » correspond à l'arrivée « du Web intelligent » qui se développe à partir du Web, entre le Logiciel Social et le Web Sémantique. Le Metaweb commence à être d'autant plus utilisé dans la mesure où nous passons d'un Web concentré sur l'information à un Web concentré sur les relations entre des choses. Ce que l'on appelle «le Web de Relation » met dorénavant l'accent sur la relation comme facteur de succès d'une activité. Comme le réseau devient de plus en plus autonome et auto-organisant, les professionnels doivent faire face à un défi: désormais, les technologies d'environnement collaboratif, soutiennent aussi bien la

06. La notion de coordination, élément de base du travail de groupe, exprime aussi le caractère dynamique du processus d'organisation du travail (De Terssac G., Lompré N., 1994, Coordination et coopération dans les organisations, Systèmes coopératifs: de la conception à la modélisation, Octarès, Toulouse). Chaque membre du groupe réalise une partie du travail seulement lorsque certaines conditions apparaissent dans l'environnement de la tâche à réaliser. On peut dire que la coordination guide l'activité. Les notions de collaboration et de coopération impliquent ainsi un lien social de nature différente entre les individus du groupe. La collaboration, au sens précis du terme, souvent employé pour le terme coopération, y compris dans les communautés scientifiques, met en jeu une qualité d'interaction particulière supérieure. Celle-ci, d'après Johnson, Johnson et Smith (1991, Cooperative learning: increasing college faculty instructional productivity, ASHE-ERIC, Washington), doit favoriser l'entraide et la confiance, être source d'encouragement, contribuer à l'établissement d'un climat non stressant, encourager le partage des ressources, viser un traitement efficace et efficient de l'information, et faciliter la remise en question des idées et raisonnements dans le but d'augmenter la qualité du travail et d'enrichir les décisions. La coopération, quant à elle, comporte une dimension d'échange négocié. l'échange négocié tend à exprimer le caractère instrumental de la coopération (observable dans les échanges effectués en contrepartie) et son caractère " politique » à travers l'influence de chaque acteur sur les règles (De Terssac G., 1992, Autonomie dans le travail, PUF, Paris) 07. Membrado M., 2008, Collaborative Working Environment State of the art, eSangathan Project, Bruxelles. 
connectivité informationnelle ${ }^{8}$ que la connectivité sociale ${ }^{9}$ Ces modalités de communication concernent particulièrement l'efficacité au travail: la virtualité peut ainsi permettre à des individus de collaborer quelles que soit les contraintes de temps et d'espace ${ }^{10}$.

Dès lors, la multiplication des outils et de leurs pratiques va permettre de passer du Web 2.0 (qui est déjà une révolution en soit pour les usagers) à des outils favorisant plus encore le knowledge management et la capitalisation des connaissances (ou, mieux encore, leur mutualisation) pour l'efficience professionnelle. L'environnement de travail collaboratif devient de facto essentiel à la performance économique de l'organisation, en créant, en modifiant et en reconfigurant les liens sociaux.

\section{Le travail restructuré}

L'élaboration de nouveaux usages liés aux modes de fonctionnement organisationnels a souvent lieu dans le cadre de l'activité professionnelle. Il devient de plus en plus courant de rencontrer des salariés mobiles ou «nomades» (essentiellement des cadres, des techniciens, des commerciaux ou des "travailleurs de la connaissance $»^{11}$ ) et en collaboration distante avec d'autres sur le terrain, auprès du client. Il s'agit par contre souvent d'une mobilité partielle car le salarié est obligé de revenir dans l'entreprise pour réaliser une partie de son travail. Souvent encore, le système d'information de l'entreprise, et plus encore l'environnement social, ne permettent pas d'être en totale mobilité.

D'une façon générale, la littérature qui traite des pratiques organisationnelles en lien avec les outils CSCW met l'accent sur la difficulté à développer une communication médiatisée plus spontanée ${ }^{12}$. Deux éléments sont ainsi particulièrement étudiés car ils ont des conséquences managériales fortes: le premier concerne l'obligation d'interrompre son travaillors d'une communication spontanée distante (Sproull, 1984 $4^{13}$; Reder \& Schwab, 19904 ; Gonzalez, \& Mark, 20045).

08. Interconnexion des infrastructures et des réseaux pour l'accès, la recherche, la diffusion et la construction d'informations

09. Interconnexion des interfaces et des réseaux permettant aux internautes d'interagir à la fois avec le contenu des pages mais aussi entre eux, notamment via le Web 2.0, faisant de ce dernier un web communautaire et interactif.

10. Demailly C., 2008, The Business Impacts of Social Networking, A White Paper in Cooperation with Early Strategies Consulting.

11. Kouloumdjian MF., Turbé-Suétens N., 2008, eSangathan White paper, A knowledge worker is a professional who works and applies his/her intellectual capacities to create, process, distribute and connect information, ideas and expertise, Bruxelles .

12. Communication qui se produit d'elle-même, librement, sans contrainte au cours d'un travail collectif

13. Sproull L. S., 1984, The nature of managerial attention. Advances in Information Processing in Organizations, 1, 9-27

14. Reder S., \& Schwab R. G., 1990, The temporal structure of cooperative activity, ACM conference on Computer-supported cooperative work, 303-316.

15. Gonzalez V. M., \& Mark G., 2004, Constant, multi-tasking craziness: managing multiple working spheres, Letters $\mathrm{CHI}, 6(1), 113-120$ 
De manière plus précise en ce qui concerne la nature de ces interruptions, le deuxième élément correspond aux variables inhérentes à ces interruptions comme les fréquences, leurs natures, leurs modalités etc. (Gillie, \& Broadbent, 1989 ${ }^{16}$; McFarlane, 199917 ; Hess, \& Detweiler, 1994 ${ }^{18}$; Cutrell, Czerwinski, \& Horvitz, 200019; Adamczyk, \& Bailey, 200420). Ces études ont bien entendues été poursuivies de façon récente car l'évolution rapide des outils collaboratifs y incite. Alors qu'elle n'en était qu'à ses débuts dans les années 2000, la « communication spontanée ", et donc de facto, le concept "d'interruption » sont aujourd'hui d'une utilisation courante en recherche (Gonzalez, \& Mark, 2004²1, Nardi, \& Kaptelinin, 200522, Belloti, Ducheneaut, Howard, \& Smith, 200323). Plus précisément, dans le cadre de la réalisation d'une activité professionnelle performante, Datchary et Licoppe $(2007)^{24}$ souligne que ce qui est donc en jeu actuellement, c'est la capacité d'un professionnel à gérer plusieurs télé-tâches à la fois (concept de "tâche associée » et de "multi-activité »), en accédant aux informations et à la communication à distance qu'il s'agisse d'une connectivité informationnelle ou d'une connectivité sociale. Cela implique clairement de prendre en considération le rapport du contexte de l'acteur à l'environnement de travail collaboratif. D'où l'intérêt de se centrer sur l'activité langagière, massivement constitutive de la communication à distance écrite ou orale.

\section{Communication et Environnements de travail collaboratif : quelles perspectives organisationnelles?}

«En parlant d'usager plutôt que d'utilisateur, il est aussi possible d'insister sur le fait que le rapport des personnes avec les outils techniques ne peut être réduit à la mise en œuvre des fonctions prévues par les concepteurs, mais que l'appropriation des technologies est une activité sociale, culturelle, économique et politique de plein droit. L'usage des technologies s'inscrit en

16. Gillie T., \& Broadbent D., 1989, What makes interruptions disruptive? A study of length, similarity and complexity, Psychological Research, 50, 243-250

17. McFarlane D., 1999, Coordinating the interruption of people in human-computer interaction, INTERACT'99, 295-303

18. Hess S. M., \& Detweiler M. C., 1994, Training to reduce the disruptive effects of interruptions, Proceedings of the Human Factors and Ergonomics Society's 38 $8^{\text {th }}$ Annual Meeting, pp. 1173-1177

19. Cutrell E. B., Czerwinski M., \& Horvitz E., 2000, Effects of Instant Messaging Interruptions on Computing Tasks, CHI 2000, Interactive Posters.

20. Adamczyk P. D., \& Bailey B. P., 2004, If Not Now, When?: The Effects of Interruption at Different Moments Within Task Execution. CHI2004, 6, 271-278

21. Gonzalez V. M., \& Mark G., 2004, Constant, constant, multi-tasking craziness: managing multiple working spheres, Letters $\mathrm{CHI}, 6(1), 113-120$

22. Nardi B., \& Kaptelinin V., 2005, Agency, Mediation, and Context: Second-Wave Interaction Design Theories, Colloque Artefact et collectif.

23. Belloti V., Ducheneaut N., Howard M., \& Smith I., 2003, Taking email to task: The design and evaluation of a task management centred email tool, $\mathrm{CHI} 2003,345-352$

24. Datchary C., Licoppe C., 2007, La multi-activité et ses appuis: l'exemple de la «présence obstinée " des messages dans l'environnement de travail, revue électronique @ctivités, volume IV numéro 1. 
effet profondément dans la vie sociale des personnes (...)»(Cardon, 2005) 25 . Dans cette optique, envisager l'appropriation des environnements collaboratifs suppose de porter la réflexion sur deux points essentiels. Il s'agit en premier lieu d'aborder le travail collaboratif en tant que support de l'activité sociale, en considérant le langage comme vecteur de coordination et d'échanges, puis d'insister sur le rapport entre TIC et interactions langagières dans ce que ces dernières apportent en termes de pratiques organisationnelles.

\section{Quand les usages des TIC développent l'activité sociale}

Pour l'analyse des environnements de travail collaboratifs, et de l'évolution de leurs usages, la notion de contexte partagé est de première importance. Nous avons dit qu'il était nécessaire, mais non suffisant, de travailler le contexte d'action collective. Néanmoins, cette dernière doit être réellement partagée, notamment au regard des informations, commentaires, opinions qui y transitent. Car il est essentiel que les usagers aient accès au même contexte d'action; il est également primordial, du point de vue cognitif, d'en avoir la même compréhension ${ }^{26}$. À cet égard, la communication langagière, qui trouve son origine au sein des différents contextes individuels, prend tout son poids. Elle favorise (Clark et Marshalli27) la coprésence linguistique (liée à tout fait évoqué oralement au cours d'une conversation verbale) et le sentiment d'appartenance à une même communauté (à chaque communauté culturelle, scientifique, familiale etc.) correspond un ensemble de connaissances partagées, linguistiques, épisodiques etc.). Or, dans l'environnement collaboratif, la pleine coprésence physique fait défaut. Ainsi, les logiques d'usage de certaines TIC ne peuvent faire l'économie de mettre en ouvre un véritable travail de transmission et de compréhension des informations interpersonnelle. Il en va de la performance cognitive individuelle et collective. Cette dernière ne se réduit pas à la transmission des représentations mentales. Elle doit être «supportée " («supported» en anglais ${ }^{28}$.) par des dispositifs techniques distribués dans l'environnement de travail (Borzeix, Cochoy, 2008) ${ }^{29}$. Elle sous-tend également les modifications et les transformations du terrain. Générer de la performance cognitive en coordonnant les actions, c'est adapter l'activité professionnelle aux nouvelles formes d'organisations de la production. Dans cette mesure, les organisations deviennent elles aussi distribuées et les

25. Cardon D ., 2005, Enjeux de mots : regards multiculturels sur les sociétés de l'information, C\&F Editions, Paris.

26. Dumazeau C., Karsenty L., 2008, Améliorer le contexte mutuellement partagé lors de communications distantes avec un outil de désignation, in Le travail humain $n^{\circ} 3$, p 225, Paris.

27. Clark H. \& Marshall R. C., 1981, Definite reference and mutual knowledge; Elements of discourse understanding, Jushi S. \& Weber (eds.), pp. 10-63

28. Nous avons choisi de garder le terme « supported » en le traduisant seulement de façon littérale car ce terme n'a pas son équivalent français. Sa traduction reste donc volontairement partielle par rapport à tout l'implicite qu'elle suppose dans son sens naturel.

29. Borzeix A., Cochoy F., 2008, Travail et théories de l'activité : vers des workspace studies, in Sociologie du travail, n50 273-286, Elsevier Masson, Paris. 
flux d'informations et les apprentissages se densifient toujours plus (Lorino, Teulier, 2005; Gherardi, 2006) ${ }^{30}$. Ainsi, les organisations, pour arriver à une logique d'usage efficace, sont-elles impérativement contraintes de développer des pratiques de knowledge management (gestion des connaissances ou ingénierie des connaissances). Celui-ci permet de mettre en œuvre un ensemble de méthodes et de techniques permettant de percevoir d'identifier, d'analyser, d'organiser de mémoriser, et de partager des connaissances entre les membres des organisations et de co-construire des savoirs internes. C'est à ce prix que les professionnels peuvent atteindre une logique d'usage optimale en s'appuyant sur l'activité langagière. D'autant que ce type d'organisation demande et amplifie de façon circulaire un fonctionnement plus en réseau, plus immatériel, avec des activités distribuées qui posent le problème de la coordination distante des actions (Veltz, 2001) ${ }^{31}$. Or, c'est bien sûr cette même communication langagière que repose la coordination des capacités relationnelles et sociales des acteurs. Dès lors, en se focalisant sur la compréhension du contexte d'action individuelle, on observe que ce type d'activité favorise dans le même temps les échanges et la reconfiguration des relations. À cet égard, la notion que développe Y.Engestrom ${ }^{32}$ est éclairante (knotworking). Elle met l'accent sur ce qu'il nomme le «travail en nœud». Il s'agit de faire coopérer des acteurs hétérogènes autour de projets plus ou moins ponctuels. Dans cette perspective, la problématique développée ci-dessus est tout à fait cohérente. Il s'agit bien ici de créer des structures relationnelles flexibles (et éventuellement temporaires) en fonction des projets de l'organisation. La coopération y est essentielle. Le langage, primordial. En d'autres termes, l'analyse de la modification de l'activité professionnelle par l'environnement collaboratif, implique une prise en compte globale, historique et culturelle de l'environnement de travail. Ce dernier va générer à son tour des changements comportementaux individuels et collectifs qui, de facto, auront une rétroaction sur les pratiques des dispositifs sociotechniques

\section{Le langage: une clé de l'appropriation}

Les recherches sur le travail et ses transformations en relation aux TIC soulignent donc l'importance de la dimension communicationnelle du travail. Plus encore, pour M. Lacoste, « le langage peut [aussi] être amené à jouer un rôle essentiel dans l'approche conjointe de la signification. L'analyse du langage permet alors de suivre, dans leurs déroulements complexes, les tentatives pour

30. Lorino P., Teulier R., 2005, Entre connaissance et organisation : I'activité collective. L'entreprise face au défi de la connaissance, Colloque de Cerisy. La Découverte, Paris.

Gherardi S., 2006, Organizational Knowledge: the texture of workplace Learning, Blackwelle Publishing. Oxford Malden, MA, Carlton.

31. Veltz P., 2001, Le nouveau monde industriel, Gallimard, Paris

32. Engestrom Y., 2008, Quand le centre se dérobe : la notion de knotworking et ses promesses, in Sociologie du travail, $n^{\circ} 50$, pages 303-330, Elsevier, Masson, Paris. 
élaborer en contexte les conditions d'une action commune ${ }^{33}$. Au cours du déroulement d'une activité,les acteurs échangent desinformations, s'organisent, signifient leurs intentions, interprètent et essayent de comprendre celles du partenaire, essayent d'atteindre un accord qui leur permettra d'élargir, ne serait-ce qu'un peu, leur champ d'autonomie et d'action. Le langage est ainsi non seulement un système social de signes pourvu d'une certaine structure mais il est encore un « instrument» (Caron, 1989) 34 . Autant, si ce n'est plus que les interactions non-verbales, les interactions langagières sont au cœur de la plupart des activités professionnelles, en présentiel ou à distance. Leur prise en compte pour l'étude fine de l'interaction sociale médiatisée s'impose, car l'activité de langage dans les situations de travail n'est pas réductible aux langages opératoires qui servent à résoudre les problèmes des acteurs engagés dans une activité collective. Elle permet l'accès à l'ensemble du contexte technique, organisationnel et social et sa compréhension.

Cesont nos pratiques sociales qui rendentle monde mutuellementintelligible. Or, l'activité de langage est présente dans nos pratiques quotidiennes. Notre compréhension partagée des situations, est due en grande partie à l'efficacité du langage qui est le moyen de contextualisation le plus efficace. Dans ce sens, Suchman (notamment) recherche dans la communication verbale le prototype d'une approche contextuelle de l'action. Ainsi, une des théories qui nous paraît toujours pertinente en termes de cadre d'analyse est la théorie de l'action située de L. Suchman (1987) ${ }^{35}$. Relevant d'une perspective sociopragmatique, elle vise à étudier l'action comme produit et résultat d'une situation sociale. L'analyse située de l'action peut tout à fait se concevoir dans le cadre des sciences sociales (et non uniquement dans le cadre des sciences cognitives). La situation y est alors étudiée en fonction de la communication verbale et de l'interaction sociale. Il s'agit de montrer que dans une situation de communication entre deux acteurs, ceux-ci rendent manifeste un «foyer d'attention ». Leur action est donc située, c'est-à-dire orientée et dépendante de l'action du destinataire. Lucy Suchman explique: «La stabilité du monde social [...] n'est pas due à une structure figée, mais aux situations d'actions qui créent et soutiennent la compréhension partagée des occasions d'interactions. Les contraintes sociales liées aux actions sont toujours identifiées comme relatives à un ensemble de contraintes spécifiques et in-reproductibles » $(1987)^{36}$. Il en est de même quand il s'agit des interactions avec les machines et la technologie. Cela suggère que l'action située hérite d'une conception de la situation, pensée à la fois en termes de communication verbale et d'interaction sociale médiatisée. L'approche située prend ainsi nettement le

33. Lacoste M., 1990, Interaction et compétences différenciées, in Réseaux, n 43, Cnet, Paris, p. 85.

34. Caron J., 1989, Précis de psycho-linguistique, PUF, Paris.

35. Suchman L., 1987, Plans and situated actions: the problem of human machine communication, Cambridge University Press, Cambridge.

36. Suchman L., 1987, Plans and situated actions: the problem of human machine communication, Cambridge University Press, Cambridge, p. 66. 
parti d'une théorie interactionniste de l'action. L'accès au contexte est donc important, mais celui-ci n'est pas donné d'avance: il est lui-même le résultat d'une interaction. Dès lors, la communication verbale offre la possibilité d'agir dans un environnement collectivement virtuel qui est à la fois le produit et le cadre de cette action. Elle contient toutes les coordonnées formées avec le contexte et représente l'immersion du locuteur dans l'acte de parole et dans les circonstances immédiates auxquelles il se réfère: l'activité de parole est énoncée dans la situation et constitue le contexte de son emploi.

Dans cette visée, la coopération devient une modalité d'action essentielle à l'interaction au sein des environnements collaboratifs. En effet, les tâches professionnelles y sont menées par des équipes dont l'activité est conditionnée par la manière dont l'information est communiquée et traitée. Une possible organisation émerge alors, dès que des acteurs ont un intérêt mutuel à coopérer. La communication établie est déjà en soi une création, un échange de valeur. Dans ce sens, on peut dire que la conversation génère l'organisation (Taylor 1993) $^{37}$. Si Taylor se place plus particulièrement dans le cadre de changement organisationnel (et l'on peut à cet égard considérer la mise en place et l'appropriation des environnements collaboratifs comme des cadres motivants de changements organisationnels), il n'en reste pas moins que c'est l'ensemble des acteurs de l'organisation et des dispositifs sociotechniques qui les entourent qui structurent l'organisation, par et dans la communication Ainsi, la construction du sens elle-même passe par la situation sociale d'interaction.

Dans cette perspective mais pris sous l'angle de la parole, du point de vue de Gramaccia $(2001)^{38}$, les actes de parole produisent aussi ce que l'on peut appeler un «illocutoire organisationnel » entre acteurs qui échangent selon les pratiques organisationnelles. Ce pourquoi il propose les éléments d'une théorie de l'illocutoire organisationnel basés sur le lien communicationnel, dont les procédés langagiers, seraient au fondement de l'organisation ellemême. L'acte de langage peut, dans certains cas, créer la structure de l'activité en même temps qu'elle l'énonce. Le terrain évoqué par l'auteur est celui $\mathrm{du}$ fonctionnement des équipes projets, dans le cadre des environnements collaboratifs: mus par la nécessité de production, les acteurs sont «obligés » de communiquer. Le besoin fondamental de cohésion des acteurs est la garantie du succès des relations humaines développées pendant la durée de vie du projet pour faciliter la convergence des savoirs, pour expliquer et partager des informations. Lillocutoire permet ainsi de donner du sens, du narratif à l'histoire de l'entreprise. Le narratif construit la cohésion entre les individus, leur fait revivre les succès et les échecs pour renforcer l'esprit d'équipe. Lillocutoire est donc essentiel à la collaboration, plus encore dans le

37. Taylor J.R., 1993, La Dynamique de Changement Organisationnel, une Théorie Conversation/Texte et ses Implications, Communication \& Organisation, $n^{\circ} 3$, mai.

38. Gramaccia G., 2001, Les actes de langage dans les organisations, Paris, L’Harmattan. 
cadre d'une activité distante médiatisée qui, comme nous l'avons rappelé plus avant, ne permet pas une réelle présence physique et mise sur la coprésence linguistique et l'appartenance à une même communauté.

\section{Éléments de conclusion}

Il est clair que l'émergence ou l'existence d'interactions verbales et de relations sociales explicites et de qualité, est un préalable à tout travail ayant pour support une communication médiatisée. Ce prérequis doit être impulsé, instauré, développé au niveau de la communication langagière. Cette dernière est, en effet, au cœur de l'appropriation des outils des environnements de travail collaboratifs. Une des difficultés à se centrer sur une telle approche dans les pratiques organisationnelles réside dans sa mise en ouvre. En effet, l'observation et l'étude de l'activité professionnelle en vue d'améliorations organisationnelles reposent soit sur l'analyse d'entretiens et d'observations - dans le cas des méthodes employées en cognition distribuée et en théories de l'activité (Halverson 2002, Theureau 2004) ${ }^{39}$ - soit sur l'enregistrement et l'analyse de données naturelles - méthodes employées par les théoriciens de l'action située. Dans tous les cas (surtout dans le dernier), les contraintes de recueil et d'analyses de données sont lourdes et l'élaboration rapide de solutions pour améliorer et faciliter les interactions d'un groupe de travail distant, difficile. Si les scientifiques peuvent, éventuellement, prendre le temps de s'immerger au sein de l'organisation (avec les difficultés épistémologiques que l'on sait), les managers quant à eux, se trouvent dans des situations d'immédiateté et d'urgence ne leur permettant guère de prendre le temps du recueil, de l'analyse des données et du réajustement par rapport à l'existant.

Plus globalement, la réflexion conduite nous incite également à ne pas souscrire à une approche déterministe des usages des technologies au sein des organisations professionnelles et à souligner la ressource que constitue l'approche en contexte d'action individuelle (comme complément essentiel à l'approche du contexte d'action collective) au travers de la communication langagière. On peut ainsi mettre l'accent sur un ensemble d'effets et de relations pour penser l'organisation d'une activité comme une dynamique, produit de rapports interpersonnels. C'est dire encore que nous considérons l'organisation d'une activité distante médiatisée non seulement comme éventuelle productrice de services (au sens économique du terme) mais également de cultures, de projets, de tensions, de régulations. Cette dynamique des relations interpersonnelles, soulignée par les approches sociopragmatiques, peut développer tout un jeu de communications, d'interactions à même de favoriser la synergie de compétences sociales et professionnelles, par la création des liens d'interdépendance entre acteurs plus

39. Halverson C., 2002, Activity theory and distributed cognition: what does CSCW need to do with theory, Computer Supported Cooperative Work 11.

Theureau, J., 2004, Cours d'action: méthode élémentaire, Octarès, Toulouse 
autonomes. Ces interdépendances supposent, cependant, que chacun participe à la relation et qu'il y ait, de ce fait, des échanges vraiment négociés au cours de la communication distante afin de réaliser une activité collaborative au plein sens du terme, fondement d'un réel construit sociocognitif. Dans cette perspective, les éléments du "carré de l'activité » (Licoppe, 2008) ${ }^{40}$, bordés par la cognition distribuée, la théorie de l'activité, l'ethnométhodologie et la sociologie des sciences et des techniques peuvent permettre de concevoir les activités professionnelles distantes au sein des environnements de travail collaboratif, comme des espaces dans lesquelles la gestion et le cadrage des tâches et leur coordination prennent toute leur valeur.

\section{BIBLIOGRAPHI€}

ADAMCZYK P. D., \& BAILEY B. P, If not now, when the effects of interruption at different moments within task execution. CHI 2004, 6, 2004, pp. 271-278

BELLOTI V., DUCHENEAUT N., HOWARD M., \& SMITH I. Taking email to task: The design and evaluation of a task management centred email tool. CHI 2003, 2003, pp. 345-352

BORZEIX A., COCHOY F., Travail et théories de l'activité: vers des workspaces studies, in Sociologie du travail, n ${ }^{\circ}$ 50273-286, Elsevier Masson, Paris, Elsevier Masson, 2008.

CARDON D., Enjeux de mots: regards multiculturels sur les sociétés de l'information, Paris, C\&F Éditions, 2005.

CARON J., Précis de psycho-linguistique, Paris, PUF, 1989.

CLARK H. \& MARSHALL R. C., Definite reference and mutual knowledge. Elements of discourse understanding. Jushi S. छ Weber (eds.), 1981, pp. 10-63

CUTRELL E. B., CZERWINSKI M., \& HORVITZ E., Effects of Instant Messaging Interruptions on Computing Tasks. CHI 2000, Interactive Posters.

DATCHARY C., LICOPPE C., « La multi-activité et ses appuis: l'exemple de la «présence obstinée » des messages dans l'environnement de travail », In: revue électronique @ ctivités, volume IV numéro 1, 2007.

DEMAILLY C., The business impacts of social networking, a white paper in cooperation with Early Strategies Consulting, 2008.

DUMAZEAU C., KARSENTY L., « Améliorer le contexte mutuellement partagé lors de communications distantes avec un outil de désignation », In: Le travail humain $\mathrm{n}^{\circ} 3$, p 225 Paris, 2008.

ENGESTROM Y., «Quand le centre se dérobe: la notion de knotworking et ses promesses », In : Sociologie du travail, ${ }^{\circ} 50$, pp 303-330, Elsevier, Masson, Paris, 2008.

FINHOLT T., SPROULL L., KIESLER S., Communication and performance, in $A d$ Hoc task groups, in Intellectual Team Work, LEA, New Jersey, 1995.

40. Licoppe C, 2008, Dans le carré de l'activité : perspectives internationales sur le travail et l'activité, in Sociologie du travail, $n^{\circ} 50$, Pages 287-300, Elsevier, Paris 
GILLIE T., \& BROADBENT D., "What makes interruptions disruptive? A study of length, similarity and complexity". In : Psychological Research n ${ }^{\circ}$ 50, 243-250, 1989.

GONZALEZ V. M., \& MARK G., CONSTANT, "multi-tasking craziness: managing multiple working spheres". Letters CHI, 6(1), 113-120, 2004.

GRAMACCIA G., Les actes de langages dans les organisations, Paris, L'Harmattan, 2001.

HALVERSON C., "Activity theory and distributed cognition: what does CSCW need to do with theory", Computer Supported Cooperative Work 11, 2002.

HESS S. M., \& DETWEILER M. C., Training to reduce the disruptive effects of interruptions. Proceedings of the Human Factors and Ergonomics Society's $38^{\text {th }}$, Annual Meeting, pp. 1173-1177, 1994.

KOULOUMDJIAN MF., TURBÉ-SUÉTENS N., eSangathan White paper, Brussels, 2008 .

LACOSTE M., «Interaction et compétences différenciées », In Réseaux, $\mathrm{n}^{\circ} 43$, Cnet, Paris, p. 85, 1990.

LICOPPE C., « Dans le carré de l'activité: perspectives internationales sur le travail et l'activité », In Sociologie du travail, n 50, Pages 287-300, Elsevier, Paris, 2008.

LORINO P., TEULIER R., « Entre connaissance et organisation: l'activité collective. L'entreprise face au défi de la connaissance », Colloque de Cerisy, Paris, La Découverte, 2005.

MCFARLANE D., Coordinating the interruption of people in human-computer interaction. INTERACT'99, pp.295-303, 1999

MEMBRADO M., Collaborative Working Environment State of the art, eSangathan Project, Bruxelles, 2008.

NARDI B., \& KAPTELININ V., "Agency, Mediation, and Context: Second-Wave Interaction Design Theories". Colloque Artefact et collectif, 2005.

REDER S., \& SCHWAB R. G., The temporal structure of cooperative activity. ACM conference on Computer-supported cooperative work, pp. 303-316,1990.

SPROULL L. S., "The nature of managerial attention. Advances”, In: Information Processing in Organizations, 1, pp. 9-27, 1984

SUCHMAN L., Plans and situated actions: the problem of human machine communication, Cambridge University Press, Cambridge, 1987.

TAYLOR J.R., La Dynamique de Changement Organisationnel, une Théorie Conversation/Texte et ses Implications, Communication $\varepsilon^{\circ}$ Organisation, n $^{\circ} 3$, mai, 1993.

VELTZ P., Le nouveau monde industriel, Gallimard, Paris, 2001.

GHERARDI S., Organizational Knowledge: the texture of workplace Learning. Blackwelle Publishing. Oxford Malden, MA, Carlton, 2006.

THEUREAU J., Cours d'action: méthode élémentaire. Toulouse, Octarès, 2004. 
Résumé : Les études sur le travail et ses transformations ont fait émerger le problème de la dimension communicationnelle du travail. Cette dernière semble essentiellement liée à l'activité langagière du collectif. Dans le cadre d'une communication (plus encore si celle-ci est médiatisée), le langage permet notamment l'accès et la compréhension du contexte technique, organisationnel et social. Le langage permet aussi l'élaboration de connaissances et de savoirs nouveaux. L'activité langagière est également un processus cognitif, psychique et affectif, où l'énonciation engage les acteurs comme individus singuliers. Enfin, l'échange verbal permet aux individus de communiquer entre eux, c'est-à-dire de transférer des données d'un individu à l'autre. Ce qui est souligné, c'est la nécessité de prendre en compte l'activité langagière dans les situations de communications distantes médiatisées. Cette activité langagière est, par essence, porteuse de processus communicationnels. Notre travail cherche donc à montrer, de façon théorique comment dans cette visée, le travail sur l'activité langagière doit être envisagée comme un outil permettant de comprendre et de mieux gérer, sur le terrain, la réalisation de l'activité.

Mots-clés : langage, communications distantes médiatisées, processus cognitifs.

\begin{abstract}
The studies on the professional work and its transformations revealed problems linked to the communicational dimension of the work. This latter seems to be essentially bound to the linguistic activity. Within the framework of communication, the language enables the access and the understanding of the technical, organizational and social context. It also eases the building of new knowledge. Furthermore, the linguistic activity is a cognitive, psychic and emotional process, in which actors are involved in all their singularity. What is underlined is the necessity of taking into account the linguistic activity in the situations of mediatised distant communications. This linguistic activity must be considered as an efficient tool to understand and to better manage professional activity.
\end{abstract}

Keywords : language, mediated communication, cognitive process 


\title{
TIC et changement organisationnel : approche par l'accompagnement
}

\author{
Isabelle Pubourdin'
}

\section{Introduction}

L'économie change, et avec elle, l'organisation. Le capital immatériel des talents, de la connaissance et des savoirs s'impose comme une composante de croissance et succède au capital matériel (Lévy, Jouyet, 2006). La production immatérielle devient le cœur de la valeur économique et la mondialisation actuelle voit l'émergence d'un troisième type de capitalisme en rupture avec les capitalismes mercantilistes et industriels: le capitalisme cognitif (MoulierBoutang, 2007). Ce changement induit un glissement des investissements vers le capital intellectuel (éducation, formation) et le travail qualifié mis en œuvre collectivement à travers les nouvelles technologies de l'information et de la communication (Tic). Ces dernières jouent alors un rôle de facilitateur de la formation et de la production du capital humain ou intellectuel. L'appropriation des connaissances et celle des usages des Tic deviennent les variables déterminantes du progrès technique et de l'innovation dans le capitalisme cognitif. Dans l'organisation éducative s'opère alors une mutation du modèle de la division du travail issu du taylorisme; l'innovation requiert la coordination de processus complexes et la coopération active des acteurs au sein de dispositifs numériques. Cette activité collective cérébrale mobilisée en réseau numérique interconnecté est autrement nommée "Concept 2.0 » (Quoniam, 2010).

Au niveau européen, la commission considère le recours aux Tic comme une modalité centrale d'édification d'un espace européen de l'enseignement. Ces orientations politiques se traduisent, au plan national, par des règlementations $\left(\mathrm{LOLF}^{2}\right.$, nouvelle loi d'orientation ${ }^{3}$ pour l'école) et s'articulent à des systèmes

01. Isabelle Pybourdin, Docteur en Sciences de l'Information et de la Communication, est membre du Laboratoire I3M à l'Université du Sud Toulon Var. Mail : Isabelle.pybourdin@univ-tln.fr

02. LOLF : loi organique relative à la Loi de Finance, découpe les fonctions de l'Etat à partir de missions, programmes et actions.

03. Loi d'orientation $n^{\circ} 2005-380$ du 23 avril 2005. 
et outils de gestion (les $\mathrm{Ent}^{4}$ ) qui s'associent à l'utilisation des Tic dans l'enseignement (Bourdin, 2008). L'objectif fixé est que les technologies soient au service d'un enseignement innovant intégrant la classe virtuelle synchrone (Gagnon, 2008). Dans l'enseignement primaire, les plans gouvernementaux RESO 2000 et $2007^{5}$ instaurent les Tic comme une composante des territoires; peu à peu les collectivités territoriales généralisent les Ent dans les établissements scolaires. Ces orientations engendrent des problématiques nouvelles qui questionnent les capacités de changement des individus et véhiculent des représentations sociales et culturelles. La confrontation de ces dernières nourrit des tensions déterminantes entre, d'une part, une politique publique d'investissements et d'orientations stratégiques du système éducatif vers des usages prescrits des Tic, et, d'autre part, les pratiques quotidiennes des acteurs au travail: entre bricolage pédagogique et pratiques de communication informelles. Dans ce contexte, l'approche communicationnelle de l'organisation (Bourdin, 2008) permet d'appréhender ensemble les logiques interpersonnelles et celles du management. L'objectif consiste à analyser comment s'articulent les situations de communication locales et les discours de l'organisation autour du concept d'accompagnement, dans un contexte de généralisation des Tic au sein des établissements scolaires.

\section{Réflexion autour de trois concepts : innovation, médiatisation, médiation}

L'innovation se distingue de l'invention (Schumpeter, 1942). Elle relève de la logique du marché ou de l'usage social alors que l'invention relève de la logique de la découverte. L'usage est ici défini par ce que Josiane Jouët (1993) désigne comme une «pratique » et ce que Laurent Thevenot (1993) désigne par le terme « usage ». L'innovation pédagogique représente alors l'ensemble des pratiques ou l'ensemble des usages conduits en présence des élèves, lesquels bousculent les routines établies et représentent une prise de risque, une forme de déviance (Alter, 2000). L'innovation ne se décrète donc pas, mais fait l'objet d'une appropriation par les acteurs au cours d'actions quotidiennes de détournement des circuits établis dans l'organisation vers de nouvelles formes de l'apprendre, ce que Pierre Musso nomme la co-innovation (2007). La médiatisation s'appréhende dans son rapport complexe avec le concept de médiation. Geneviève Jacquinot (2005) nous rappelle ainsi que si ces deux concepts ont la même origine étymologique, ils restent cependant distincts sans être opposés puisqu'ils ne correspondent pas aux mêmes fonctions: la médiation renvoie à l'action du médiateur, tandis que la médiatisation s'entend au travers de la fonction de médiatiser et représente une action indirecte. Ainsi la médiation est une instance qui articule le singulier et le collectif, le sujet singulier et la communauté à laquelle il appartient (Lamizet, 1994). Elle

04. Ent : Environnement numérique de travail.

05. RESO 200 et 2007 : Plans gouvernementaux Pour une République numérique dans la société de l'information. 
se définit alors comme ce qui permet la représentation et la communication à l'autre d'idées, de savoirs, de sentiments, d'impulsions qui nous appartiennent grâce à une culture, c'est-à-dire grâce à un système commun de représentations. L'appropriation des Tic en milieu scolaire semble ainsi liée à la construction d'usages endogènes et à la capacité de faire émerger un espace de médiation entre l'acteur et la technique.

\section{Le dispositif numérique le " P'tit journ@I "}

Au sein desétablissements scolaires, l'avènement du Web 2.0 etla généralisation des équipements informatiques marquent l'émergence de nouveaux moyens de production de contenus et de partage des connaissances: les sites contributifs. Ces derniers autorisent le développement de dispositifs pédagogiques fondés sur l'idée d'un travail collaboratif à distance (Lundgren-Cayrol, Henri, 2003) ou d'une communication et d'un apprentissage instrumentés en réseau (Jocair, 2006). Le « P'tit journ@1» (Pybourdin, 2005) est l'un d'entre eux. Ce dispositif de médiatisation de contenus concerne une institution éducative du premier degré. Il s'agit d'un journal contributif en ligne.

Articulé autour d'une interface de publication collective en ligne, SPIP ${ }^{6}$, c'est à la fois un dispositif pédagogique et un projet de communication ${ }^{7}$ s'adressant aux écoles élémentaires d'une circonscription. Les participants collaborent à la publication d'un magazine en ligne, dont les écrits font l'objet d'une négociation interne entre classes avant d'être publiés sur Internet. Chacun des rédacteurs s'inscrit dans une unité espace/temps particulière, quand il se connecte sur l'interface numérique. Deux sortes de communication sont alors envisagées: une communication synchrone via la messagerie instantanée dans le cas ou les deux classes partagent la même unité espace/temps; une communication asynchrone via les commentaires rédigés au bas des articles, dans le cas contraire. Ces situations de communication sur l'interface engendrent du lien social entre une vingtaine de classes localisées dans trois communes. Le propos ici défendu s'articule autour du questionnement suivant: la pratique de la communication médiatisée en réseau et du magazine en ligne favorise-t-elle le développement de nouvelles compétences communicationnelles (Miège, 2007) en lien avec la production de contenus sur Internet?

\section{Méthodologie}

Des méthodes d'inspiration ethnographiques sont développées afin d'observer l'appropriation ${ }^{8} \mathrm{du}$ " P'tit journ@1 » dans quatre établissements

06. SPIP : Système de publication pour l'Internet partagé

07. Le projet de communication place les enseignants et leurs élèves en situation de travail coopératif et collaboratif en ligne.

08. Le concept d'appropriation est entendu ici au sens de Serge Proulx (Breton, Proulx, 2002) à savoir une maîtrise cognitive et technique minimale du dispositif technique, une intégration significative de l'usage de cette technologie dans le quotidien des acteurs, la possibilité de développer des gestes de création par lesquels l'usage de l'objet technique fait émerger de la nouveauté dans le vie de l'usager. 
scolaires, auprès de vingt enseignants participants. Le corpus d'observations participantes complètes conduites pendant trois années scolaires et d'entretiens compréhensifs (Kaufmann, 2001) sont traités dans le respect d'une approche qualitative. L'observation du chercheur est issue de sa propre intégration sur le terrain de la recherche sous le statut de Chercheur-ActeurImpliqué (Pybourdin, 2008). Cette posture interroge la neutralité objective du chercheur et sa capacité de distanciation. Cependant, les méthodes qualitatives retenues lui fournissent un cadre pour l'analyse et l'interprétation des données. L'ethnométhodologie et l'analyse qualitative du recueil de données participent d'une approche compréhensive des phénomènes. Cette démarche comporte des moments de saisie intuitive des significations dont les faits humains et sociaux sont porteurs à partir d'un effort d'empathie opéré par le chercheur.

Les résultats de cette recherche montrent que l'appropriation des technologies dans la classe relève plus de la continuité d'activités préexistantes (Chaptal, 2007), testées et efficaces, que de la rupture vers une nouvelle forme de l'apprendre, soit une innovation pédagogique.

\section{Discussion}

L'approche empirique montre que la production d'articles en classe fait majoritairement l'objet d'une démarche de production d'écrits collectifs ou individuels. Les élèves rédigent un article à plusieurs mains, en présentiel et selon des objectifs assignés par le maître, ou bien, un article individuel faisant l'objet d'une évaluation portant sur des critères d'apprentissage. Ils le saisissent ensuite sur l'interface et le proposent en relecture à leurs partenaires. Ce mode d'organisation pédagogique est propre au paradigme d'enseignement (Tardif, 1998) qui privilégie le collectif et la simultanéité, l'acquisition de connaissances et de compétences. Toutefois, il a été remarqué une séquence pédagogique relevant du paradigme d'apprentissage (Tardif, 1998) au cours de laquelle les élèves étaient acteurs et responsables de la construction des savoirs.

L'innovation résiderait dans une véritable pratique collaborative du «P'tit journ@1» permettant de traiter à plusieurs classes un thème d'étude déterminé et de confronter les approches diverses de ce sujet au cours d'une communication éducative instrumentée en réseau. "Chaque participant peut s'inscrire, à la fois dans ses propres productions et face à celles des autres, dans l'étude et dans la délibération, tout en participant d'un collectif de pensée élaboré par le travail en retour sur ces productions. " (Sensevy \& al, 2005 : 329). Au cours de l'étude empirique, ce genre de pratique ne fait pas encore l'objet d'une appropriation par les élèves et les enseignants. En effet, le «P'tit journ@1 » est censé fonctionner comme un organe de presse avec un comité éditorial composé d'élèves et un comité de rédaction formé d'adultes. Pour hiérarchiser les articles et coordonner la publication en ligne, l'utilisation du forum est 
d'une grande importance et suppose que les participants mutualisent, débattent et collaborent, à distance, à des réalisations originales. Cependant, le forum d'échange s'apparente davantage à une équipe lato sensu, un groupe d'échanges qu'à des équipes stricto sensu avec une coordination des pratiques (Perrenoud, 1997 : 26-33). Dans les faits, les enseignants proposent l'abandon de cette forme de l'échange dès la co-évolution (Fichez, 2006) du dispositif sociotechnique (Flichy, 2003) à l'issue de la première publication.

Par ailleurs, l'interface « P'tit journ@1 » privilégie le partenariat entre classes autour de la rédaction d'articles et l'interaction des lecteurs avec les rédacteurs quand ces textes sont publiés sur Internet. Au terme de la première année scolaire de mise en place du «P'tit journ@1 » soixante et onze articles sont publiés par les classes participantes. Ces dernières ont utilisé le forum attaché aux articles pour déposer leurs commentaires pendant les phases d'échanges entre classes partenaires, avant la publication en ligne. Les enseignants ont pris part au réseau d'échanges réciproques de savoirs, le Rers (HebertSuffrin, 2001), afin de mutualiser des compétences en rapport avec les Tic. Cependant, les échanges cessent au sein de la communauté des participants dès que les textes sont publiés sur Internet. La mise en ligne semble marquer la fin du processus de coopération et l'apparition de lecteurs silencieux. JeanMarc Turban (2005: 342) constate ainsi que « le très faible taux d'adhésion aux listes de diffusion pédagogiques du primaire invite à ne pas surestimer l'impact de ces réseaux sociotechniques sur la corporation des maîtres du primaire». En fait, l'auteur constate qu'assez peu de maîtres, et surtout de maîtresses, parviennent à consacrer quotidiennement plusieurs dizaines de minutes à fréquenter les forums ou listes de discussion. Les enseignants sont-ils prêts et suffisamment formés pour faire un usage professionnel de l'informatique, qui ne se résume pas seulement à la préparation de la classe mais intègre aussi la coopération et la collaboration via les réseaux. S'il est admis, à la suite de Georges Vignaux (2003:103), que les Tic offrent les conditions favorables à l'émergence de collectifs intelligents au sein de "réseaux d'information où cognition individuelles et cognition collective s'interpénétreront ", la recherche montre que la logique d'usage (Perriault, 1989) des acteurs commande le succès ou l'échec de l'intégration des Tic au sein du système éducatif. Il semble que les politiques publiques, qui conduisent à la généralisation des Tic dans les établissements scolaires, s'inscrivent dans une logique diffusionniste (Rogers, 1983), une «marche forcée » (Puimato, 2007) vers le tout technique. Cette logique instrumentale privilégie les équipements au détriment des usages et crée une tension entre une grande quantité de matériels installés dans les établissements et la faiblesse de leurs usages par les enseignants en situation pédagogique (Chaptal, 2007). Ce qui fait défaut sont «les méthodes pour utiliser (les Tic), les intégrer dans un cours, évaluer leur utilisation par les élèves » (Moeglin, 2005 : 208). Cette démarche conduit peu à peu à la construction de la fracture par les usages dans l'enseignement (Pybourdin, 2009). 


\section{L'accompagnement : une médiation de la communication vers l'acculturation aux Tic}

Les Tic n'échappent pas à la distinction de l'endogène et de l'exogène. Le plan de généralisation des Tic au sein du système éducatif relève de décisions publiques exogènes à la communauté éducative (Belisle \& al., 2007; Barats, 2006, Bourdin, 2008). Ces projets imposés par le haut peuvent contraindre les individus et contrarier les dynamiques locales (Pouts-Lajus, 2005). Il s'avère donc nécessaire de considérer les besoins de la communauté éducative et sa capacité à intégrer les Tic dans ses pratiques quotidiennes. Le problème de l'acculturation progressive aux nouveaux outils est posé: comprendre comment chaque enseignant s'approprie peu à peu les Tic et développe des usages en présence des élèves. Comme on ne décrète pas l'usage des Tic, il s'agit de s'intéresser à la question du changement individuel et de son accompagnement.

Pour obtenir le changement au niveau de l'individu, trois démarches sont possibles. La première est celle des psychothérapies qui permettent aux patients de parvenir au mieux être et de découvrir la transformation de soi. La seconde propose de changer de cadre de référence (Watzlawick, 1972, 1980) et la troisième considère le changement comme l'apprentissage de nouveaux comportements. Cet apprentissage est élémentaire quand il consiste en une simple modification de comportement; il devient plus décisif quand il repose sur une modification des connaissances et des représentations. Dans certains cas, ce changement correspond à une simple adaptation, dans d'autres, il sollicite des capacités de réflexion et déclenche des réactions émotionnelles conséquentes (Emery, 2000). Ce coût émotionnel peut générer une résistance au changement car "en amont du savoir, du vouloir et du pouvoir se situent les émotions. » (Emery, 2000). Cette approche nous permet de penser qu'un accompagnement des enseignants peut favoriser la remise en cause des routines établies et l'apparition de pratiques pédagogiques innovantes intégrant les Tic.

L'accompagnement est ici considéré comme la volonté de mettre en scène un tiers, une parole, un acte de langage, soit un intermédiaire entre la technique et le social, les Tic et les enseignants. Selon Bruno Devauchelle (2007), la formation des enseignants est le frein le plus important au développement des Tic en milieu scolaire et universitaire. Nombre d'entre eux utilisent l'ordinateur pour eux-mêmes, mais rares sont ceux qui l'intègrent dans leur pratique. "Plutôt que d'une formation stricto-sensu, ils ont surtout besoin d'un accompagnement. "Celui ci consiste à installer dans les établissements des personnes dont le rôle est de « rendre les enseignants capables de » et non pas de faire à leur place. Ces personnes ressources sont en fait des « passeurs» (Bonniol, 1996) dont la mission est de rendre l'Autre compétent par l'intermédiaire d'une médiation technique et sociale (Vygotsky, 1997 ; Jouët, 1993 ; Peraya, 2000) et d'une médiation de la communication (Lamizet, 1994). 
Pour penser le concept d'accompagnement, la théorie interactionniste de Lev Vygotsky et le modèle socioconstructiviste de l'apprentissage sont convoqués. L'acte d'apprendre est alors considéré comme un acte fondé sur les interactions sociales et la relation d'aide. Il s'agit d'une médiation sociale et technique et d'une médiation de la communication. Ainsi, l'accompagnement consiste à emboîter le pas à quelqu'un, partager son chemin, l'épauler juste un temps. L'accompagnateur est alors l'intermédiaire qui introduit de la distance et du lien quand il permet à celui qui avance de regarder son chemin et à celui qui peine de se reprendre et quand il oriente les uns vers les autres. L'enjeu consiste ainsi à instaurer «une logique de la communication" (Watzlawick \& $a l, 1972$ ) favorable à l'accompagnement des pratiques d'intégration des Tic et à user, de ce fait, du langage, du texte, du cotexte et du contexte pour agir sur les représentations et construire un sens partagé dans l'interaction et l'inter énonciation.

Pour construire le sens dans l'interaction, il faut considérer que l'apprentissage est couplé à l'immersion de la personne dans la culture et dans les interactions sociales, de sorte que la connaissance soit située dans un contexte social et culturel avant de faire l'objet d'une appropriation individuelle. Les connaissances sont indissociables des contextes dans lesquels elles s'élaborent. L'accompagnateur est alors ce médiateur qui communique dans la Zone proximale de développement $(\mathrm{Zpd})$ et aide, par le langage, les enseignants qui désirent acquérir des compétences tant techniques que pédagogiques en rapport avec l'utilisation des Tic en classe. La cognition est ainsi socialement située et l'activité mentale est associée à l'environnement culturel et social dans lequel elle se réalise et aux ressources qu'il fournit. Car apprendre à utiliser un ordinateur, c'est s'intégrer dans une culture où les connaissances et les compétences prennent un sens en lien avec la manière de les utiliser selon les contextes. C'est un processus d'acculturation par lequel celui qui apprend doit entrer dans une communauté de pratique, dans un contexte socioculturel qui octroie du sens aux activités d'apprentissage. Ceci suppose la mise en place d'activités authentiques et signifiantes dans un contexte déterminé où l'accompagnement consiste à garantir un climat d'entraide et de collaboration.

Le partage du sens dans l'inter énonciation participe de ce que JeanJacques Boutaud (1998) nomme le paradigme de la signification. Après le paradigme du signal (Shannon et Weaver, 1948) et celui du système (Winkin, 1981), il traite l'information comme un phénomène de sens. Il considère le sens comme un construit, socialement situé dans l'inter énonciation, les jeux illocutoires et l'inter discursivité. Le sens du message s'élabore à partir de la représentation du réel par des subjectivités en interaction, à partir de ce qui prend valeur de vérité pour chacun des sujets en interaction. L'espace de communication est donc l'espace d'un sens négocié dans lequel le processus d'intercompréhension porte moins sur le dit, le contenu, que sur la manière de le 
dire, la relation. Dans les situations communicationnelles d'accompagnement, les travaux d'Erwing Goffman $(1973,1974)$ fournissent un cadre conceptuel qui complète les notions de texte (le verbal, la para verbal et le non verbal), de co-texte (l'environnement discursif) et de contexte (le cadre et la relation). Le sens se négocie, est construit dans un contexte particulier, dans des situations d'interénonciation.

L'accompagnement des enseignants dans un contexte situé permet de guider le changement des pratiques pédagogiques par la médiation de la communication. Cet accompagnement facilite la médiation technique et sociale par laquelle l'acculturation et l'appropriation de la technique s'élaborent.

\section{Conclusion : Deux formes complémentaires de communication en organisation}

Le «P'tit journ@1» ne peut donc être réduit à un «texte » (Taylor, 1993) que les usagers doivent lire, mais il permet de construire un certain nombre de pratiques innovantes favorisées par l'accompagnement. Le concept d'accompagnement assure une médiation de la communication, soit une dialectique du singulier au collectif (Lamizet, 1994). Il permet de penser l'émergence du changement dans les actes, au cours de la "conversation» (Taylor, 1993) d'acteurs coopérant au sein d'un "illocutoire organisationnel » (Gramaccia, 2001). Les interactions ainsi générées permettent à chacun de nouer des liens de coopération sur la base d'actes de langage prononcés, en contexte, par les locuteurs disposant d'une légitimité symbolique pour agir. Elles deviennent des jeux de relations, des processus réflexifs, implicites et actifs qui relient les partenaires par la force de l'illocutoire et les actes d'engagement (Bernard, 2004). Dans ce contexte se développe une logique de communication informelle, invisible et opaque entre acteurs de la coopération, entre «communicants de peu» (Bernard, 1997) portés par des finalités communes.

L'accompagnement est alors conceptualisé au moyen d'une modélisation, considérée "comme dispositif pragmatique et empirique d'aide à la réflexion.» (Le Moënne, 2006 : 29). Deux formes de communication organisationnelle se distinguent: la communication linéaire, instituée et hiérarchique, et la communication circulaire, inventée et informelle (Pybourdin, 2008).

La communication linéaire est une communication normée et stabilisée faisant référence au modèle «émetteur - récepteur ». Elle contient les discours du management et les valeurs de l'organisation. Dans le système éducatif, cette communication porte sur la diffusion des textes de lois, des décrets et des circulaires qui déterminent un cadre d'action contraint pour les enseignants et se centre sur les seuls éléments explicites des situations et notamment sur 


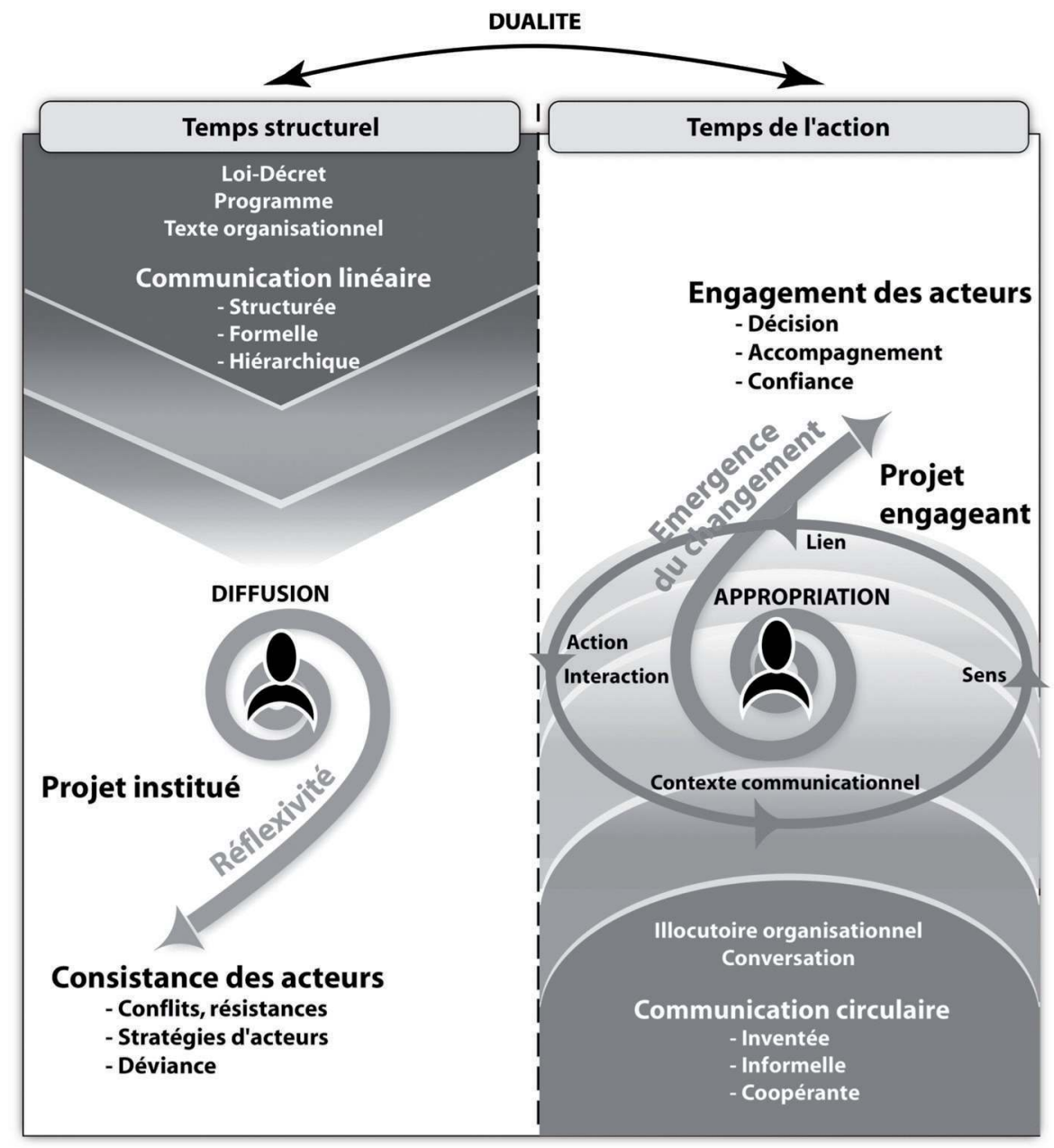

\begin{tabular}{|c|c|}
\hline COMPORTEMENTALISME & SOCIO-CONSTRUCTIVISME \\
\hline CONTEXTE CONTRAINT, AUTORITE & CONTEXTE DE LIBERTE, AUTO-ORGANISATION \\
\hline $\begin{array}{l}\text { FONCTIONNALISME - THEORIE DES SYSTEMES } \\
\text { STRUCTURALISME }\end{array}$ & SUBJECTIVISME - HERMENEUTIQUE \\
\hline L'EXPLIQUER & LE COMPRENDRE \\
\hline \multicolumn{2}{|c|}{ PRINCIPE DIALOGIQUE } \\
\hline
\end{tabular}

Figure 1: Deux formes de la communication au micro niveau de l'organisation (Pybourdin, 2008) 
le verbal, écrit ou oral. Ainsi, dans le cadre de la généralisation des Tic dans le système éducatif, les enseignants reçoivent les messages de l'institution en ce qui concerne les cadres de certification (B2i) et le socle de compétences à valider en fin de cycle 3. Ces informations sont reçues avec une quasi-passivité puisqu'ils ne peuvent agir sur le contenu des programmes publiés au Bulletin officiel. Ils peuvent cependant interpréter ce «texte organisationnel» (Taylor, 1993) et les contraintes qu'il porte, se risquer à la sanction par des stratégies de déviance (Alter, 2000), des stratégies de pouvoir (Crozier, Friedberg, 1977) et des phénomènes de résistance au changement (Crozier, 1984) dans une réflexivité correspondant à l'actualisation des contraintes de l'organisation en fonction de leurs objectifs et de leurs enjeux d'acteurs (Giddens, 1987).

La communication circulaire est imprévisible et orientée vers l'intercompréhension et le sens. Elle contribue au développement de la coopération et de la confiance (Gramaccia, 2007). Dans le cadre de l'accompagnement, cette communication s'inscrit dans une problématique du lien, du sens, de l'action et de l'interaction qui valorise l'intentionnalité des enseignants au cours de la "conversation» (Taylor, 1993). Le rituel de co-présence (Gramaccia, 2001), les différentes attitudes, les codes culturels, les interactions en contexte et le maintien du lien social fondent un code de comportements individuels qui favorise l'accompagnement et l'interprétation $\mathrm{du}$ «texte» dans une dynamique ascendante portant l'émergence du changement.

L'accompagnement des enseignants vers un usage collaboratif des Tic dans la classe dépend plus d'une forme circulaire de la communication que d'une forme linéaire portant les injonctions du management. L'articulation des logiques organisationnelles aux logiques individuelles se révèle fondamental pour impliquer l'individu, le considérer comme un acteur autonome et responsable (Castoriadis, 1975), susceptible de manifester ses intentions au sein de l'organisation et d'éprouver des sentiments d'appartenance. 


\section{BIBLIOGRAPHIE}

ALTER, N., L'innovation ordinaire, Paris: PUF, 2000, 284 p.

BARATS, C., «Discours et pratiques sur les Tice dans un établissement », In: Jocair, juillet 2006, Université de Picardie Jules Verne: Amiens, 2006, p. 177-203.

BÉLISLE, C., ROSADO, E., Usages des Tice en éducation: leurre ou levier? Les dossiers de l'ingénierie éducative, septembre 2007, Hors série, p. 37- 46.

BERCOT, R., «Les réseaux de santé: une configuration sociétaire », In:De SAINTLAURENT-KOGAN, A-F., METZGER, J-L. (Dir), Où va le travail à l'ère numérique, Mines Paris: Presse ParisTech, 2007, p. 51-70, (Coll. Sciences sociales).

BERNARD, F., "La communication de changement, vers une heuristique de l'induction ", Communication Eं organisation, 1997, n 12, p 303-337.

BERNARD, F., JOULE, R-V., "Lien, sens et action: vers une communication engageante ", Communication $\mathcal{E}^{\circ}$ organisation, 2004, n 24 , p. 333-345.

BONNIOL, J.J, La passe ou l'impasse: le formateur est un passeur, Les cabiers de l'année, Université de Provence, 1996, n 1, p. 9-25.

BOURDIN, S, «Les TICE comme dispositif: contribution à une approche communicationnelle du changement organisationnel ", In:_I3M-Sfsic, Les dispositifs de médiation organisationnelle, technologique et symbolique dans la communication des organisations, Nice: 2008, p. 290-295.

BOUTAUD, J-J, Sémiotique et communication: du signe au sens, Paris: L'Harmattan, 1998, 318 p.

BRETON, P., PROULX, S., L'explosion de la communication à l'aube du XXI' siècle. Paris: Éditions La Découverte, 2002, 389 p.

CASTORIADIS, C., L’institution imaginaire de la société, Paris: Seuil, 1975, 503 p. (Coll. Esprit).

CHAPTAL, A., Paradoxe des usages des Tice, Dossiers de l'ingénierie éducative, septembre 2007, Hors-série, p. 73-91.

CROZIER, M., FRIEDBERG, E., L'acteur et le système, Paris: Le Seuil, 1977, 437 p.

CROZIER, M., On ne change pas la société par décret, Paris: Hachette-Pluriel, 1984/1979, 310 p.

Devauchelle, B., Pour un accompagnement formatif de proximité à l'intégration des Tic. Disponible sur www.brunodevauchelle.com (29/10/07).

EMERY, J-L., « Le rôle des émotions », In: Sciences Humaines, 2000, Hors série $\mathrm{n}^{\circ} 28$, p. 14-16.

FICHEZ, E., « L'industrialisation de la formation », In: Terminal, 2000, n 83, p. 65-72.

FICHEZ, E., « Plate-forme collaborative « Postnuke » : dynamique de co-construction et de co-évolution », In: Jocair, juillet 2006, Université de Picardie Jules Verne: Amiens, 2006, p. 27-50.

FLICHY, P, L'innovation technique: vers une nouvelle théorie de l'innovation, Paris: La Découverte, 2003, 250 p. 
GAGNON, N, «Former les enseignants au numérique », In Colloque International L'université à l'ère numérique (CIUEN), décembre 2008, Bordeaux.

GIDDENS, A., La constitution de la société, Paris: PUF, 1987, 474 p.

GOFFMAN, E., La mise en scène de la vie quotidienne, Paris: Éditions de Minuit, 1973, $256 \mathrm{p}$.

GOFFMAN, E., Les rites d'interaction, Paris: Éditions de Minuit, 1974, 230 p.

GRAMACCIA, G., Les actes de langage dans les organisations, Paris: L'Harmattan, 2001, 286 p, (coll. Communication des organisations).

GRAMACCIA, G., "Les actes de langage dans les organisations par projet », In Bonneville, L. et Grosjean, S (Dirs.), Repenser la communication dans les organisations, Paris: L'Harmattan, 2007, p 51-86.

HEBERT-SUFFRIN, C., Echangeons nos savoirs, Paris: Syros, 2001, 200 p.

JACQUINOT, G., et MONNOYER L., "Avant propos, il était une fois ", In: Hermès, $\mathrm{n}^{\circ}$ 25, édition 1999, 2005, p. 9-14.

JOCAIR, Premières journées communication et apprentissage instrumentés en réseau, Université de Picardie Jules Verne: Amiens, 2006, 543 p.

JOUËT, J, « Pratiques de communication et figures de la médiation », Réseaux, 1993, $\mathrm{n}^{\circ} 60$.

KAUFMANN, J-C., L'entretien compréhensif, 2e ed., Paris: Armand Colin, 2001 1996, 127 p. (Coll. Sociologie 128, n 137).

LAMIZET, B., « Médiation et communication », In: Chappaz G. (Dir.), Comprendre et construire la médiation, Université de Provence: Crdp Marseille, 1994, p. 135-150.

LE MOËNNE, C., "Considérations sur les méthodes de recherche en communications organisationnelles ", In: Bouzon, A.\& Meyer, V. (Dirs.), La communication organisationnelle en question, Paris: L'Harmattan, 2006, p. 15-30.

LÉVY, M., JOUYET, P., «L'économie de l'immatériel, la croissance de demain », Rapport de la commission sur l'économie de l'immatériel: Paris, 2006.

LINARD, M., "La distance en formation: une occasion de repenser l'acte d'apprendre », In: Davies, G., Tinsley, D. (Ed.), Accès à la Formation à Distance, Clés pour un Développement Durable, Genève: FIM Erlangen, p. 46-55.

LUNDGREN-CAYROL, K, HENRI, F., Apprentissage collaboratif à distance, Quebec: PU du Québec, 2003, 184 p.

METZGER, J-L., « Elaboration et mode d'usage d'une typologie des collectifs ", In: DE SAINT LAURENT-KOGAN, A-F., METZGER, J-L. (Dirs), Où va le travail à l'ère numérique, Mines Paris: Presse ParisTech, 2007, p. 23-49, (Coll. Sciences sociales).

MIEGE, B., La société conquise par la communication, Grenoble: PUG, 2007, 235 p.

MOEGLIN, P, Outils et médias éducatifs: une approche communicationnelle, Grenoble: PUG, 2005, 296 p.

MOULIER-BOUTANG, Y., Le capitalisme cognitif, Paris: Éditions Amsterdam, 2007, 315 p. (Coll. Multitudes/Idées)

MUSSO, P, PONTHON, L, SEULLIET, E, Fabriquer le futur 2: l'imaginaire au service de l'innovation, Village Mondial, Paris: Pearson Education France, 2007, 307 p. 
PERAYA, D., Internet, un nouveau dispositif de médiation des formes des savoirs et des comportements, Genève: TEFCA, 2000.

PERRENOUD, P, Réfléchir ou agir ensemble? , Éducateur, 1997, n² 12, p 8-11.

PERRET-CLERMONT, A-N; NICOLET, M., Interagir et connaître, enjeux et régulation dans le développement cognitif, Paris: Delval, 1988, 351 p.

PERRIAULT, J., La logique de l'usage: essai sur les machines à communiquer, Mayenne: Flammarion, 1989, 255 p.

POUTS-LAJUS, S., "Classification des Ent et comparaisons internationales ", Dossiers de l'ingénierie éducative - Environnements numériques de travail, 2005, p. 25-35.

PUIMATTO, G., De l'outil à l'usage, Les dossiers de l'ingénierie de l'éducation, septembre 2007, Hors-série, p. 15-33.

PYBOURDIN, I, 2005, Un P'tit journ@l à la page, Médialog, 2005, n 53, p 4-7.

PYBOURDIN, I., Appropriation des technologies de l'information et de la communication: le cas d'un "projet engageant" conduit au sein d'une institution éducative du premier degré. Approche communicationnelle, accompagnement, médiations, Th. Sic. Toulon: Université du Sud Toulon Var, juin 2008, 454 p.

PYBOURDIN, I, «Politiques publiques: construction de la fracture par les usages dans l'enseignement », In: Les Cahiers du Numérique, 2009, volume 5-n¹/2009, p. 195222.

QUONIAM, L, « Du Web 2.0 au concept 2.0 », In: Les Cahiers du numérique, Paris: Hermès-Lavoisier, 2010, 198 p.

ROGERS, E., Diffusion of innovation, New York: The Free Press, 1983, (3e édition).

SCHANNON, C.E., WEAVER, W., La théorie mathématique de la communication, Paris: Cepl,p. 1948-1975 pour l'édition française.

SCHUMPETER, J.A, Capitalisme, socialisme et démocratie, trad. Franç. Paris: Payot, 1942/ 1972, 417 p.

SENSEVY, G; KUSTER, Y; HELARY, F, «Le forum débat: un dispositif d'apprentissage collaborative en formation initiale des enseignants ", In: Distances et savoirs, 2005, vol. 3-4/2005, p. 311-330.

TARDIF, J, Intégrer les nouvelles technologies de l'information: quel cadre pédagogique, Paris: ESF, 1998, 127 p.

TAYLOR, J, « La dynamique de changement organisationnel », In: Communication \& organisation, $1993, \mathrm{n}^{\circ}$ 3, p. 51-93.

THEVENOT, L., «Essai sur les objets usuels. Propriétés, fonctions, usages », In: Raisons pratiques, 1993, n 4, p 85-111.

TURBAN, J-M, «Listes de diffusion pour enseignants du premier degré », In: Distances et savoirs, 2005, vol. 3-4/2005, p. 331-355.

VIGNAUX, G., « L'autoformation, élément pivot de la cognition naturelle, In: Albero, B. (Dir), L'autoformation et l'apprentissage supérieur, Paris: Hermes Lavoisier, 2003, p 93-103.

VYGOTSKY, L, Pensée et langage, 3e édition, Paris: La dispute, 1997, 537 p. (1933, $1^{\text {re édition) }}$ 
WATZLAWICK, P., HELMICK BEAVIN, J., JACKSON, D., Une logique de la communication, Paris: Le Seuil, 1972, 280 p.

WATZALWICK, P., Le langage du changement, Paris, Le Seuil, 1980, 185 p.

WINKIN, Y., Anthropologie de la communication. De la théorie au terrain, Paris: Le Seuil, 2001, 332 p. (coll. Points essais).

Résumé : Cette contribution propose d'analyser comment s'articulent les situations de communication locales et les discours de l'organisation à travers le concept d'accompagnement. Ce dernier est envisagé comme une médiation de la communication permettant d'appréhender ensemble les logiques interpersonnelles et les logiques managériales.

Mots-clés : Tic, usage collaboratif, organisation, accompagnement, médiations, interactions, inter énonciation.

Abstract : This article aims to analyse how the local situations of communication and the speeches of organization are structured through the concept of follow up. This is regarded as a mediation of communication allowing to analyse both the interpersonal logics and the managerial ones.

Keywords : Tic, networking, organization, follow up, interaction and mediation 